\title{
Identification of time-series differentially expressed genes and pathways associated with heart failure post-myocardial infarction using integrated bioinformatics analysis
}

\author{
XUEFEI LI ${ }^{1-3 *}, \mathrm{BIN} \mathrm{LI}^{4 *}$ and HONG JIANG ${ }^{1-3}$ \\ ${ }^{1}$ Department of Cardiology and ${ }^{2}$ Hubei Key Laboratory of Cardiology, Renmin Hospital of Wuhan University; \\ ${ }^{3}$ Cardiovascular Research Institute, Wuhan University, Wuhan, Hubei 430060; ${ }^{4}$ Department of Orthopedic Surgery, \\ Zhongnan Hospital of Wuhan University, Wuhan, Hubei 430071, P.R. China
}

Received August 22, 2018; Accepted March 13, 2019

DOI: $10.3892 / \mathrm{mmr} .2019 .10190$

\begin{abstract}
Heart failure (HF) secondary to acute myocardial infarction (AMI) is a public health concern. The current study aimed to investigate differentially expressed genes (DEGs) and their possible function in HF post-myocardial infarction. The GSE59867 dataset included microarray data from peripheral blood samples obtained from HF and non-HF patients following AMI at 4 time points (admission, discharge, and 1 and 6 months post-AMI). Time-series DEGs were analyzed using R Bioconductor. Functional enrichment analysis was performed, followed by analysis of protein-protein interactions (PPIs). A total of 108 DEGs on admission, 32 DEGs on discharge, 41 DEGs at 1 month post-AMI and 19 DEGs at 6 months post-AMI were identified. Among these DEGs,
\end{abstract}

Correspondence to: Professor Hong Jiang, Department of Cardiology, Renmin Hospital of Wuhan University, 238 Jiefang Road, Wuhan, Hubei 430060, P.R. China

E-mail: jianghwurm@163.com

${ }^{*}$ Contributed equally

Abbreviations: AMI, acute myocardial infarction; AK5, adenylate kinase 5; CXCL8, C-X-C motif chemokine ligand 8; DEGs, differentially expressed genes; DAVID, Database for Annotation, Visualization and Integrated Discovery; FADS2, fatty acid desaturase 2; FMN1, formin 1; GPR15, G-protein coupled receptor 15; GO, gene ontology; HF, heart failure; HUFA, highly unsaturated fatty acids; KEGG, Kyoto Encyclopedia of Genes and Genomes; IL, interleukin; JDP2, Jun dimerization protein 2; LRRN3, leucine-rich repeat neuronal protein 3; LVEF, left ventricular ejection fractions; MAPK, mitogen-activated protein kinase; NT-proBNP, N-terminal pro-brain natriuretic peptide; PPI, protein-protein interaction; PTGS2, prostaglandin-endoperoxide synthase 2; RNASE1, ribonuclease A family member 1; STRING, search tool for the retrieval of interacting genes; THBS1, thrombospondin 1; TNF $\alpha$, tumor necrosis factor $\alpha$

Key words: heart failure post-myocardial infarction, bioinformatics, time-series, differentially expressed genes, protein-protein network
4 genes were downregulated at all the 4 time points. These included fatty acid desaturase 2 , leucine rich repeat neuronal protein 3, G-protein coupled receptor 15 and adenylate kinase 5. Functional enrichment analysis revealed that these DEGs were mainly enriched in 'inflammatory response', 'immune response', 'toll-like receptor signaling pathway' and 'NF- $\kappa \beta$ signaling pathway'. Furthermore, PPI network analysis revealed that $\mathrm{C}-\mathrm{X}-\mathrm{C}$ motif chemokine ligand 8 and interleukin $1 \beta$ were hub genes. The current study identified candidate DEGs and pathways that may serve important roles in the development of HF following AMI. The results obtained in the current study may guide the development of novel therapeutic agents for HF following AMI.

\section{Introduction}

The prevalence of heart failure (HF) is $>23$ million worldwide at present and its number is continuing to increase (1). Although the management of HF has improved in the past 3 decades, the 5-year mortality rate of patients with HF remains high at $\sim 50 \%$ (2). A common cause of HF is acute myocardial infarction (AMI), which often signals the onset of cardiac dysfunction that may progress to HF (3). This progression is dependent on the extent of myocardial damage, recurrent ischemia, the development of myocardial stunning and hibernation, ventricle remodeling and chronic neuroendocrine stimulation (4). The incidence of HF among patients hospitalized for an AMI varies among studies, ranging between 14 and 36\% (5-7). This highlights the requirement for early and effective prediction tools and subsequent intervention following AMI.

Biomarkers such as brain-type natriuretic peptide and $\mathrm{N}$-terminal pro-brain natriuretic peptide (NT-proBNP) are associated with ventricle remodeling and the development of $\mathrm{HF}$, and are widely applied in the clinical diagnosis and prognosis of HF $(8,9)$; they have a fair prognostic value for $\mathrm{HF}$ in patients with acute coronary syndromes (10). However, these biomarkers lack specificity, as they are elevated in patients with renal failure, primary aldosteronism, congestive HF and thyroid disease (11). Genome-wide gene expression profiling has been extensively used for screening new potential biomarkers for the diagnosis and/or prediction 
of disease severity, such as breast cancer and gastric adenocarcinoma $(12,13)$, as well as the identification of novel drug targets (14). Maciejak et al (15) have previously utilized this approach to identify new biomarkers that may have predictive value for HF following AMI, including ribonuclease A family member 1 pancreatic (RNASE1), formin 1 (FMN1) and Jun dimerization protein 2 (JDP2). However, the dynamic expression changes of the key genes and associated biological processes in the development of HF following AMI have not been fully elucidated.

The present study used the microarray data of the GSE59867 dataset deposited by Maciejak et al (15) to identify time-series differentially expressed genes (DEGs) and pathways associated with HF following AMI using comprehensive bioinformatics methods. The time-series DEGs and their associated functions and pathways were analyzed. The protein-protein interaction network (PPI) of these DEGs was subsequently constructed and hub genes were identified. Results from the current study may provide novel insights into potential prognostic and therapeutic targets for HF following AMI.

\section{Materials and methods}

Data sources. The gene expression profile data of GSE59867 were downloaded from the Gene Expression Omnibus database (GEO; www.ncbi.nlm.nih.gov/geo). The GeneChip Human Gene 1.0 ST Array (Affymetrix; Thermo Fisher Scientific, Inc.) platform was used. The dataset contained samples from 111 patients with ST-elevation myocardial infarction at 4 time points: i) Admission, the day when AMI was confirmed; ii) discharge, 4-6 days post-AMI; iii) 1 month post-AMI; and iv) 6 months post-AMI. The dataset included samples at the 4 aforementioned time points from 46 control patients that had stable coronary artery disease on the day of admission, and no history of myocardial infarction (MI). Among the 111 patients with ST-elevation MI, 9 patients were diagnosed with HF and 8 patients were considered no to have HF on the basis of the first and fourth quartiles of plasma NT-proBNP level and left ventricular ejection fractions (LVEF) at 6 months post-AMI. The mean \pm standard deviation of the age of the HF and non-HF patients was $60.1 \pm 14.3$ and $51.8 \pm 7.2$, respectively. There were no significant differences in mean age, sex, body mass index or history of hypertension, diabetes, smoking, hypercholesterolemia, MI and AMI. Additionally, there was no significant difference in aspirin, clopidogrel, $\beta$-blockers, angiotensin-converting-enzyme inhibitors and statins taken by the two groups of patients. The percentage of HF and non-HF patients taking diuretics was 77.8 and $12.5 \%$, respectively. The level of NT-proBNP was $918.3 \pm 848.5$ and $62.0 \pm 14.1 \mathrm{pg} / \mathrm{ml}$ in $\mathrm{HF}$ and non-HF patients respectively, while the level of LVEF was $39.3 \pm 8.4$ and $66.8 \pm 1.9 \%$, respectively. There were significant differences in these three variables between the two groups (Table I). A total of 8 patients with HF and 6 non-HF patients were followed across the aforementioned 4 time points and were used for subsequent analysis.

Data preprocessing. The raw data were quantile normalized using the robust multiarray average in the Affy package (www. bioconductor.org/packages/release/bioc/html/affy.html) (16) in $\mathrm{R}$ Bioconductor. The probes were converted into gene symbols according to probe annotation information. If multiple probes corresponded to the same gene symbol, the median was calculated as the gene expression value of the gene. The probes that matched with multiple genes were deleted. Additionally, the expression values of the genes with unknown specific functions were removed.

DEGs analysis. The DEGs in peripheral blood samples from HF patients at different time points compared with non-HF patients were identified using the Limma package (www.bioconductor.org/packages/release/bioc/html/limma.html) (17) in R Bioconductor. A fold-change of gene expression ratio of $>1.5$ and $\mathrm{P}<0.05$ were used as the cut-off criteria. The hierarchical clustering analysis of these DEGs was performed using Genesis (genome.tugraz.at) (18). Additionally, a Venn diagram was created using Venny software (version 2.1; bioinfogp.cnb. csic.es/tools/venny/index.html).

Functional enrichment analysis. To explore the potential biological processes and pathways that may be involved in patients with post-AMI HF, the Database for Annotation, Visualization and Integrated Discovery (DAVID; david. ncifcrf.gov.uk) (19) was used to perform Gene Ontology (GO) annotation and Kyoto Encyclopedia of Genes and Genomes (KEGG) pathway analysis for the DEGs. $\mathrm{P}<0.05$ was used as the threshold value, and the number of genes enriched in each pathway was $\geq 2$. The differences in biological processes between the non-HF and HF patients at different time points were investigated.

PPI construction. PPI networks are used to organize all protein-coding genes into a large network that provides a better understanding of the functional organization of the proteome (20). The Search Tool for the Retrieval of Interacting Genes (STRING; string-db.org/cgi/input.pl) (21) database provides information regarding predicted and experimental interactions of proteins in a given cell. In the present study, the DEGs were mapped into the STRING database to identify significant protein pairs with a combined score of $>0.4$. The PPI network was subsequently constructed using Cytoscape software, version 3.6.1 (www.cytoscape.org) (22). The nodes with a higher degree of interaction were considered as hub genes. Additionally, cluster analysis for identifying significant function modules with a degree cutoff $>3$ in the PPI network was performed using the Molecular Complex Detection plug-in (23) in Cytoscape. GO biological process enrichment analysis for the DEGs in modules of the PPI network was performed using the Biological Networks GO plug-in (24) in Cytoscape. $\mathrm{P}<0.05$ was considered to indicate a statistically significant difference.

Statistical analysis. All continuous data are expressed as the mean \pm standard deviation while categorical data are presented as frequencies and percentages, and both were analyzed using SPSS software, version 19.0 (IBM Corp.). Each experiment was repeated three times. A Student's t-test was used to compare the continuous data between the two patient groups. Categorical data between the two patient groups were compared using Fisher's exact test. $\mathrm{P}<0.05$ was considered to indicate a statistically significant difference. 
Table I. Demographic characteristics of HF and non-HF patients.

\begin{tabular}{|c|c|c|c|}
\hline Variables & HF patients $(n=9)$ & Non-HF patients $(\mathrm{n}=8)$ & P-value \\
\hline Age, years (mean \pm standard deviation) & $60.1 \pm 14.3$ & $51.8 \pm 7.2$ & 0.147 \\
\hline Male sex, $\mathrm{n}(\%)$ & $6(66.7)$ & $7(87.5)$ & 0.576 \\
\hline Body mass index, $\mathrm{kg} / \mathrm{m}^{2}$ (mean \pm standard deviation $)$ & $26.8 \pm 3.1$ & $25.6 \pm 1.6$ & 0.323 \\
\hline Hypertension, n (\%) & $3(33.3)$ & $1(12.5)$ & 0.576 \\
\hline Diabetes, n $(\%)$ & $2(22.2)$ & $1(12.5)$ & $>0.999$ \\
\hline Smoking, n (\%) & $3(33.3)$ & $5(62.5)$ & 0.347 \\
\hline Hypercholesterolemia, n (\%) & $5(55.6)$ & $4(50.0)$ & $>0.999$ \\
\hline Previous MI, n (\%) & $0(0.0)$ & $0(0.0)$ & NA \\
\hline AMI, n (\%) & $8(88.9)$ & $3(37.5)$ & 0.106 \\
\hline Aspirin, n (\%) & $9(100.0)$ & $8(100.0)$ & NA \\
\hline Clopidogrel, n (\%) & $8(88.9)$ & $8(100.0)$ & $>0.999$ \\
\hline$\beta$-blockers, n (\%) & $9(100.0)$ & $8(100.0)$ & NA \\
\hline ACE inhibitors, n (\%) & $9(100.0)$ & $8(100.0)$ & NA \\
\hline Statins, n $(\%)$ & $9(100.0)$ & $8(100.0)$ & NA \\
\hline Diuretics, n $(\%)$ & $7(77.8)$ & $1(12.5)$ & 0.015 \\
\hline NT-proBNP, pg/ml (mean \pm standard deviation) & $918.3 \pm 848.5$ & $62.0 \pm 14.1$ & $<0.001$ \\
\hline LVEF, \% (mean \pm standard deviation) & $39.3 \pm 8.4$ & $66.8 \pm 1.9$ & 0.001 \\
\hline
\end{tabular}

$\mathrm{HF}$, heart failure; ACE, angiotensin-converting enzyme; AMI, acute myocardial infarction; MI, myocardial infarction; NT-proBNP, N-terminal pro-brain natriuretic peptide; LVEF, left ventricular ejection fraction.

\section{Results}

Identification of candidate DEGs in HF following AMI. A total of 108 DEGs (46 upregulated and 62 downregulated genes) on admission, 32 DEGs (16 upregulated and 16 downregulated genes) on discharge, 41 DEGs (22 upregulated and 19 downregulated genes) at 1 month post-AMI and 19 DEGs (2 upregulated and 17 downregulated genes) at 6 months post-AMI were identified between peripheral blood samples obtained from patients with $\mathrm{HF}$ and non-HF patients (Fig. 1). Among these DEGs, 4 genes were identified at each of the 4 time points (Fig. 2A), namely, fatty acid desaturase 2 (FADS2), leucine-rich repeat neuronal protein 3 (LRRN3), G-protein coupled receptor 15 (GPR15) and adenylate kinase 5 (AK5). Compared with those of samples obtained from non-HF patients, the gene expression values of the aforementioned 4 genes were significantly downregulated at the different time points following AMI in patients with $\mathrm{HF}(\mathrm{P}<0.05$, Fig. 2B).

Functional enrichment analysis of biological processes. The potential functions and pathways of the time-series DEGs were analyzed using DAVID. The dynamic change of biological processes in the development of $\mathrm{HF}$ following AMI is presented in Fig. 3. The results revealed 18 biological processes on admission, 12 biological processes on discharge, 17 biological processes at 1 month post-AMI and 3 biological processes at 6 months post- AMI. Compared with the biological processes at 6 months post-AMI, which is considered the stable phase (15), the 'inflammatory response' and 'immune response' were enriched on admission. On discharge, the biological processes were mainly enriched in response to stress, such as 'response to drug', 'response to muscle stretch', 'cellular response to fatty acid' and so on. The biological processes at 1 month post-AMI were mainly enriched in the regulation of cells and cytokines, including 'positive regulation of smooth muscle cell proliferation' and, 'positive regulation of endothelial cell migration', 'cellular response to growth factor stimulus' and 'positive regulation of vascular endothelial growth factor production'. Notably, biological processes involved in the 'inflammatory response' were enriched in the first three time points.

Functional enrichment analysis of KEGG pathways. The KEGG pathways of the DEGs were analyzed using DAVID. A total of 7 KEGG pathways were significantly enriched on admission, including 'cell adhesion molecules', 'cytokine-cytokine receptor interaction', 'T cell receptor signaling pathway', 'intestinal immune network for IgA production', 'hematopoietic cell lineage', 'amoebiasis' and 'malaria' (Fig. 4). A total of 7 KEGG pathways were significantly enriched on discharge, including 'toll-like receptor signaling pathway', 'pertussis', 'salmonella infection', 'rheumatoid arthritis', 'Chagas disease', 'hepatitis B' and 'antigen processing and presentation'. A total of 3 KEGG pathways were significantly enriched at 1 month post-AMI and included the 'NF- $\kappa \beta$ signaling pathway', 'bladder cancer' and 'malaria'. Finally, 1 KEGG pathway, 'nitrogen metabolism', was significantly enriched at 6 months post-AMI.

PPI network analysis. A total of 32 nodes and 44 protein pairs on admission, 11 nodes and 10 protein pairs on discharge, 8 nodes and 12 protein pairs at 1 month post-AMI, and 2 nodes and 1 protein pair at 6 months post-AMI, with a PPI score of $>0.4$ based on the STRING database, were obtained (Fig. 5A). The results revealed that interleukin $1 \beta$ (IL1 $\beta$; degree, 10), 
A Admission

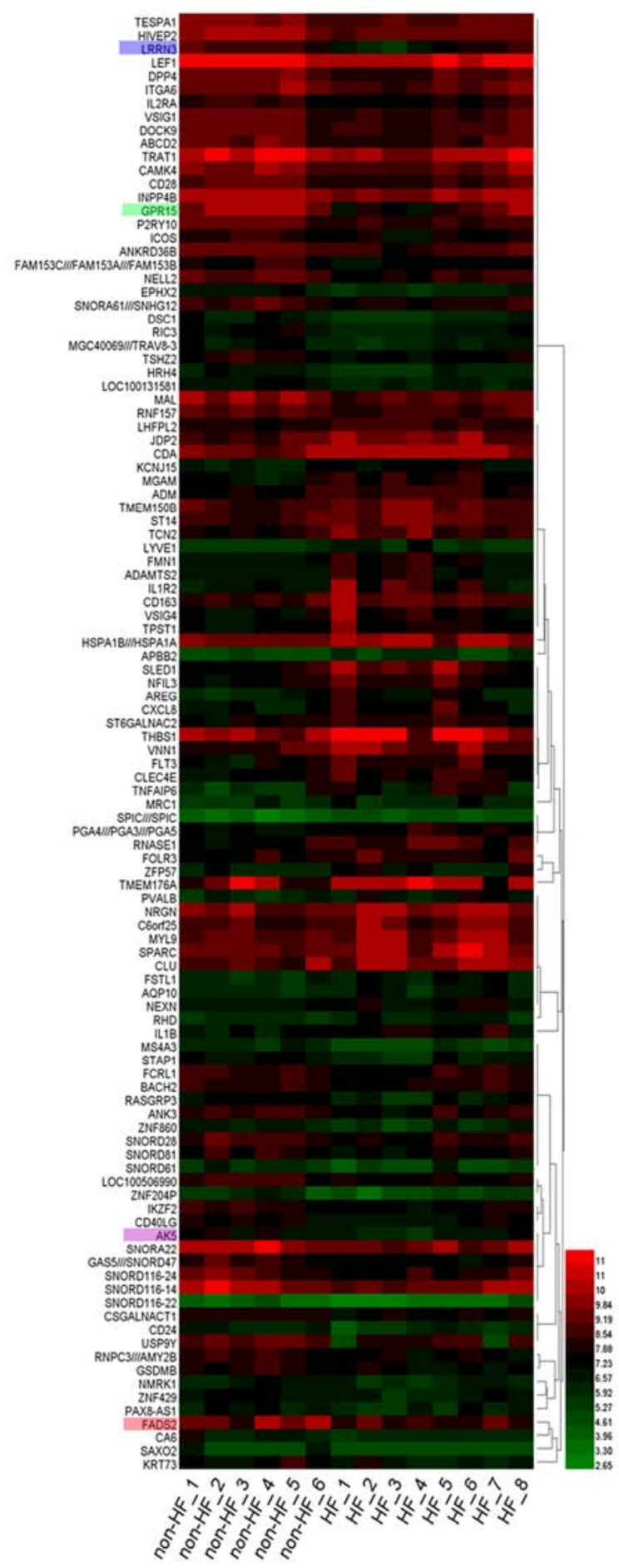

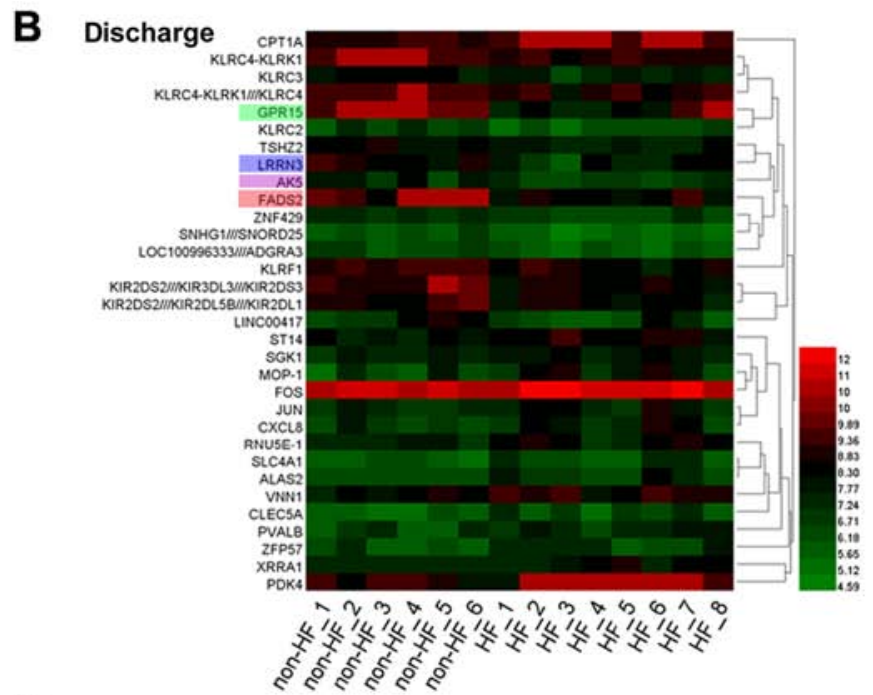
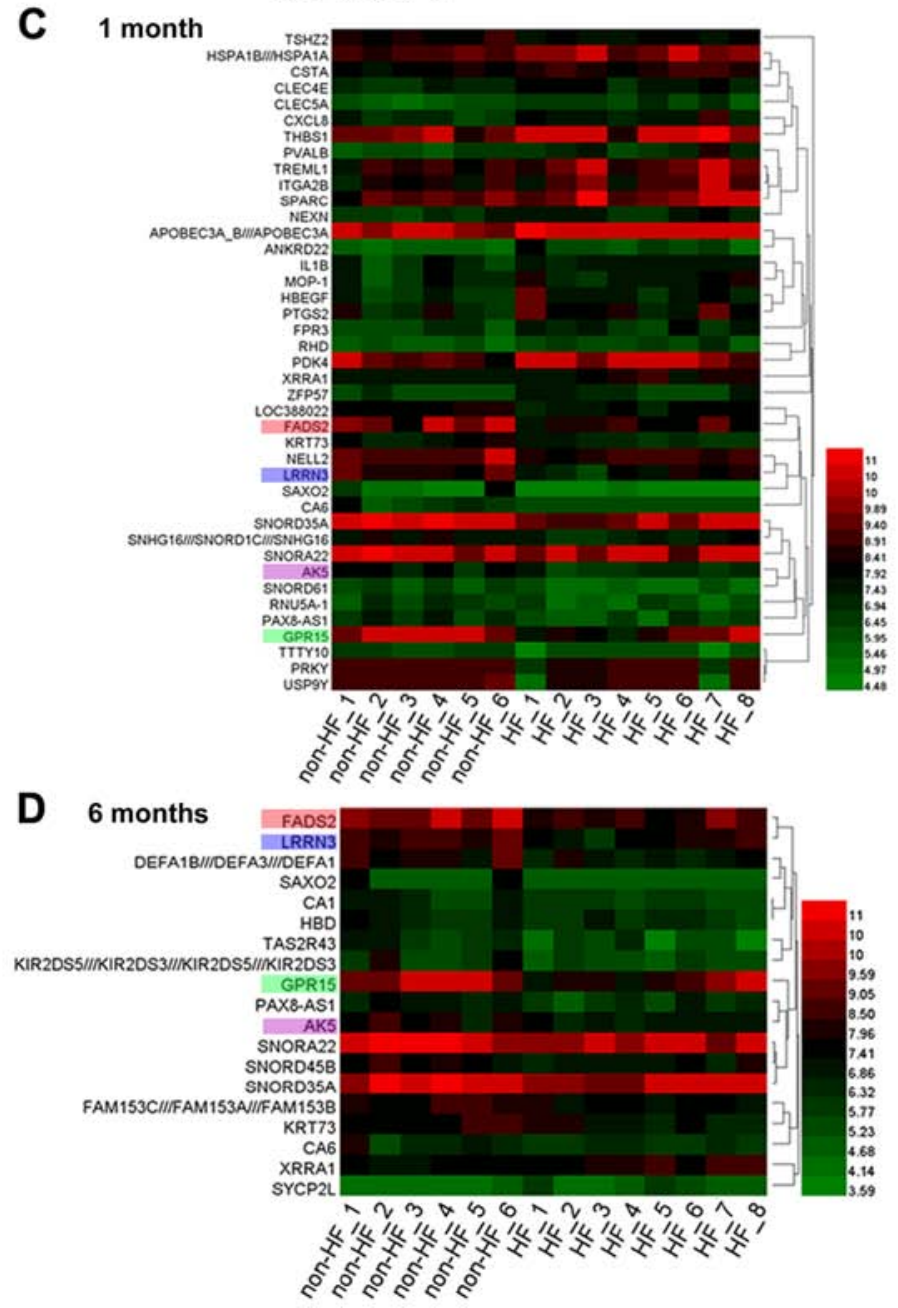

Figure 1. Heat map of the DEGs identified in HF and non-HF patients at different time points. DEGs identified (A) on admission, (B) on discharge, (C) at 1 month post-AMI and (D) at 6 months post-AMI. Green represents lower expression, and red represents higher expression. A set of 4 genes was identified at each time point. Each gene is presented by a different color: i) FADS2 (pink); ii) LRRN3 (blue); iii) GPR15 (green); and iv) AK5 (purple). DEGs, differentially expressed genes; HF, heart failure; AMI, acute myocardial infarction; FADS2, fatty acid desaturase 2; LRRN3, leucine-rich repeat neuronal protein 3; GPR15, G-protein coupled receptor 15; AK5, adenylate kinase 5.

C-X-C motif chemokine ligand 8 (CXCL8; degree, 10) and thrombospondin 1 (THBS1; degree, 7) on admission, Fos proto-oncogene AP-1 transcription factor subunit (degree, 4), Jun proto-oncogene AP-1 transcription factor subunit (degree, 3) and CXCL8 (degree, 2) on discharge, and CXCL8 (degree, 6), THBS1 (degree, 4) and prostaglandin-endoperoxide synthase 2 (PTGS2; degree, 4) at 1 month post-AMI were the top three hub genes in the first three time points. Notably, CXCL8 was the hub gene identified in all of the first three time points. Additionally, carbonic anhydrase (CA1; degree, 1) 
A

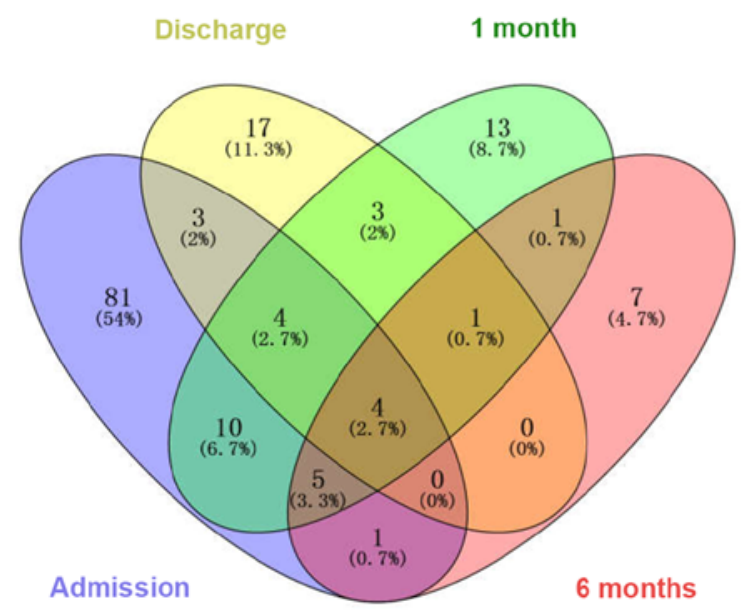

B
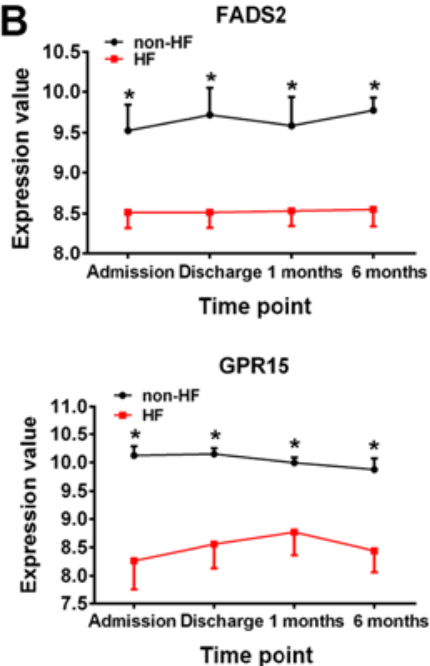

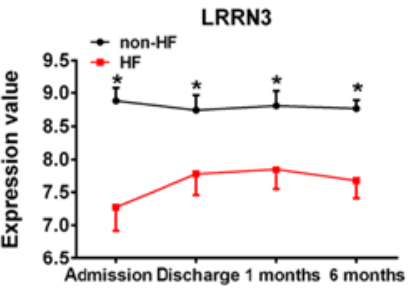

Time point

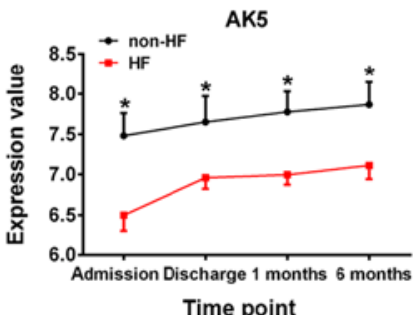

Figure 2. Identification of DEGs in HF following AMI. (A) Venn diagram of the DEGs. Different colours represent different time points: i) Blue, admission; ii) yellow, discharge; iii) green, 1 month post-AMI; and iv) pink, 6 months post-AMI. (B) The changes in expression levels of the genes FADS2, LRRN3, GPR15 and AK5 in HF and non-HF patients at the different time points. ${ }^{*} \mathrm{P}<0.05$ vs. HF group. DEGs, differentially expressed genes; HF, heart failure; AMI, acute myocardial infarction; FADS2, fatty acid desaturase 2; LRRN3, leucine-rich repeat neuronal protein 3; GPR15, G-protein coupled receptor 15; AK5, adenylate kinase 5.

and hemoglobin subunit $\delta$ (HBD; degree, 1) were identified at 6 months post-AMI.

Additionally, 1 module network on admission and 1 module network at 1 month post-AMI were identified. Nodes, including CXCL8, IL1 $\beta$, CD40 ligand, tumor necrosis factor $\alpha(\mathrm{TNF} \alpha)$-induced protein 6 and interleukin 2 receptor subunit $\alpha$, were highlighted on admission (Fig. 5B). The highlighted nodes at 1 month post-AMI were CXCL8, IL1 $\beta$, PTGS2 and heparin binding EGF-like growth factor. IL1 $\beta$ and CXCL8 were enriched in the 2 modules. However, no significant biological processes and pathways were enriched in these module networks.

\section{Discussion}

Maciejak et al (15) identified 3 upregulated genes on admission following AMI, including RNASE1, FMN1 and JDP2, which were associated with the increase of NT-proBNP and the decrease of LVEF at 6 months post AMI. The study demonstrated that these 3 genes may have prognostic value for patients with HF. These 3 upregulated genes were identified in the present study when comparing patients with HF patients with non-HF patients on admission. Additionally, the present study identified 4 genes, namely FADS2, LRRN3, GPR15 and AK5, which were downregulated on admission, on discharge and at 1 and 6 months post-AMI. This suggested that these four genes may serve roles in the progression of HF following AMI and may be potential therapeutic targets for preventing the development of $\mathrm{HF}$ following AMI.

Additionally, by analyzing the gene expression profiles in peripheral blood samples obtained from patients with $\mathrm{HF}$ patients and non-HF patients at different time points, the present study identified a series of DEGs enriched in pathways of the inflammatory response, immune response, the toll-like receptor signaling pathway and the $\mathrm{NF}-\kappa \beta$ signaling pathway.
CXCL8 and IL1 $\beta$ were identified as hub genes in the PPI network.

FADS2 is a member of the fatty acid desaturase family, which are important enzymes in fatty acid metabolism (25). FADS2, also known as $\Delta$-6-desaturase, is one of the 3 main desaturases in humans, and is required for the synthesis of highly unsaturated fatty acids (HUFA) (25). Previous studies indicated that reduced serum concentrations of omega-3 and omega-6 HUFA were associated with the progression of HF (26-28). Alter et al (29) suggested that increased ventricular wall stress and a reduced LVEF were associated with decreased docosahexaenoic acid, a HUFA. Furthermore, a clinical study demonstrated that dietary supplementation with the HUFA eicosapentaenoic and docosahexaenoic acids may prevent sudden cardiac death, acute coronary syndrome and HF (30). The current study revealed that the expression level of FADS2 was downregulated in patients with $\mathrm{HF}$ when compared with that in patients with non-HF during the entire follow-up period, suggesting that FADS2 may serve an important role in the development of HF following AMI.

As a member of the neuronal leucine-rich repeat family, LRRN3 is a type I transmembrane protein (31). Fukamachi et al (32) reported that LRRN3 may enhance the phosphorylation of mitogen-activated protein kinase (MAPK) and therefore activate its signaling pathway in COS-7 cell lines, originally isolated from the kidney of the African green monkey. Previous studies demonstrated that the MAPK signaling pathway is implicated in the development of HF (33-35). Zhang et al (34) revealed that the MAPK signaling pathway regulated cardiomyocyte apoptosis during post-infarction HF in mice. Fei et al (35) revealed that the MAPK signaling pathway contributed to angiotensin II-induced cardiac fibrosis in rats. Therefore, LRRN3 may mediate the development of HF following AMI via the MAPK signaling pathway and its downstream effectors. 
A Admission

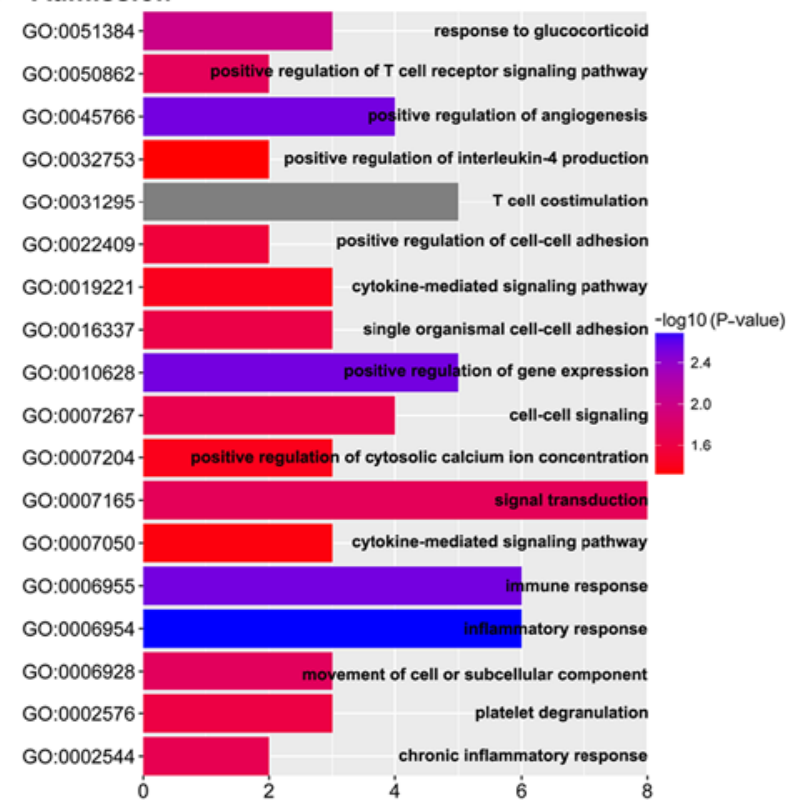

C 1 month

GO:0071363- cellular response to growth factor stimulus

GO:0050715- positive regulation of cytokine secretion

GO:0048661. positive regulation of mooth muscle cell proliferation

GO:0045766 positive regulation of angiogenesis

GO:0045429- positive regulation of nitric oxide biosynthetic process

GO:0042346- positive regulation of NF-kappaB import into nucleus

GO:0031622- positive regulation of fever generation
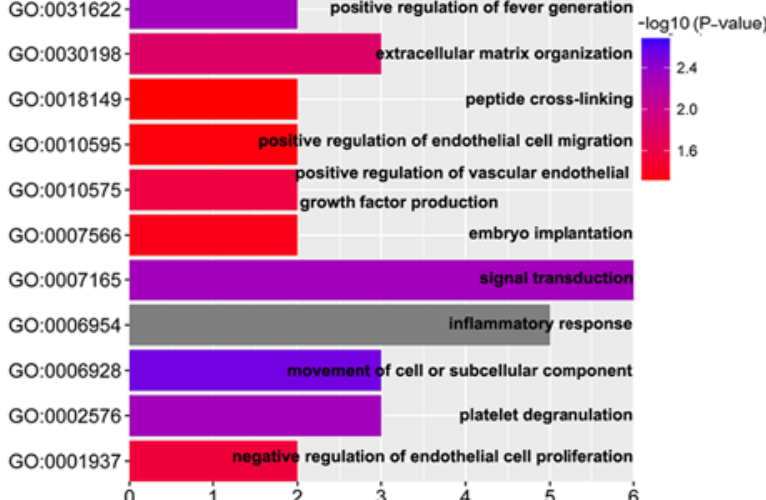

\section{B Discharge}

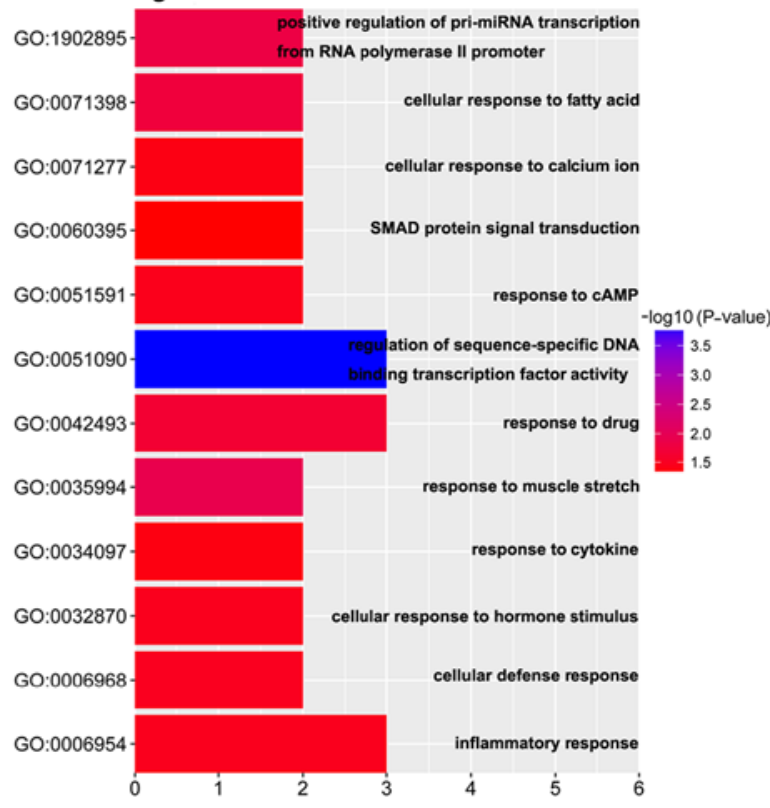

D 6 months

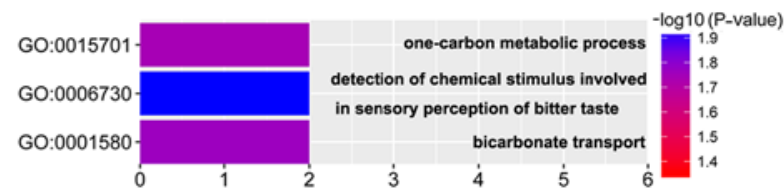

Figure 3. Significant GO biological process terms enriched at different time points. Differentially expressed genes identified (A) on admission, (B) on discharge, (C) at 1 month post-AMI and (D) at 6 months post-AMI. AMI, acute myocardial infarction; GO, Gene Ontology.

GRP15 is an orphan receptor member of the G-protein coupled receptor family that was initially identified as a co-receptor for the human immunodeficiency virus (36). GPR15 was required for the recruitment of $\mathrm{T}$ helper $17, \mathrm{~T}$ helper 1 effector and regulatory $\mathrm{T}$ cells in a colitis model in mice (37). Koks et al (38) reported that the expression level of GPR15 was upregulated in the blood of smokers and served a potential role in chronic inflammatory pathologies. Pan et al (39) demonstrated that GPR15 binds the fifth epidermal growth factor-like region of thrombomodulin and mediates angiogenesis and protection of vascular endothelial cells by activating extracellular signal-regulated kinase and increasing production of anti-apoptotic protein and NO. Thrombomodulin did not protect vascular endothelial cells isolated from GPR15 knockout mice from tacrolimus-induced vascular injury (39). Based on the results obtained in the aforementioned studies, GRP15 may serve a role in the progression of HF following AMI.

AK5 belongs to adenylate kinase family (40). Adenylate kinases catalyze the reversible nucleotide phosphoryl exchange reaction AMP+ATP $\rightarrow 2 \mathrm{ADP}$, and thus control the cellular energy supply $(40,41)$. Unlike the other members of the adenylate kinase family, AK5 is a cytosolic isoform highly expressed in the brain, and it serves an important role in neurogenesis and neuronal-specific metabolism (40). Lai et al (42) reported that the complex of AK5 and copine VI was involved in epileptogenesis, which was caused by neuronal hyperexcitability. However, to the best of our knowledge, the association between AK5 and HF has not been investigated. The decreased 


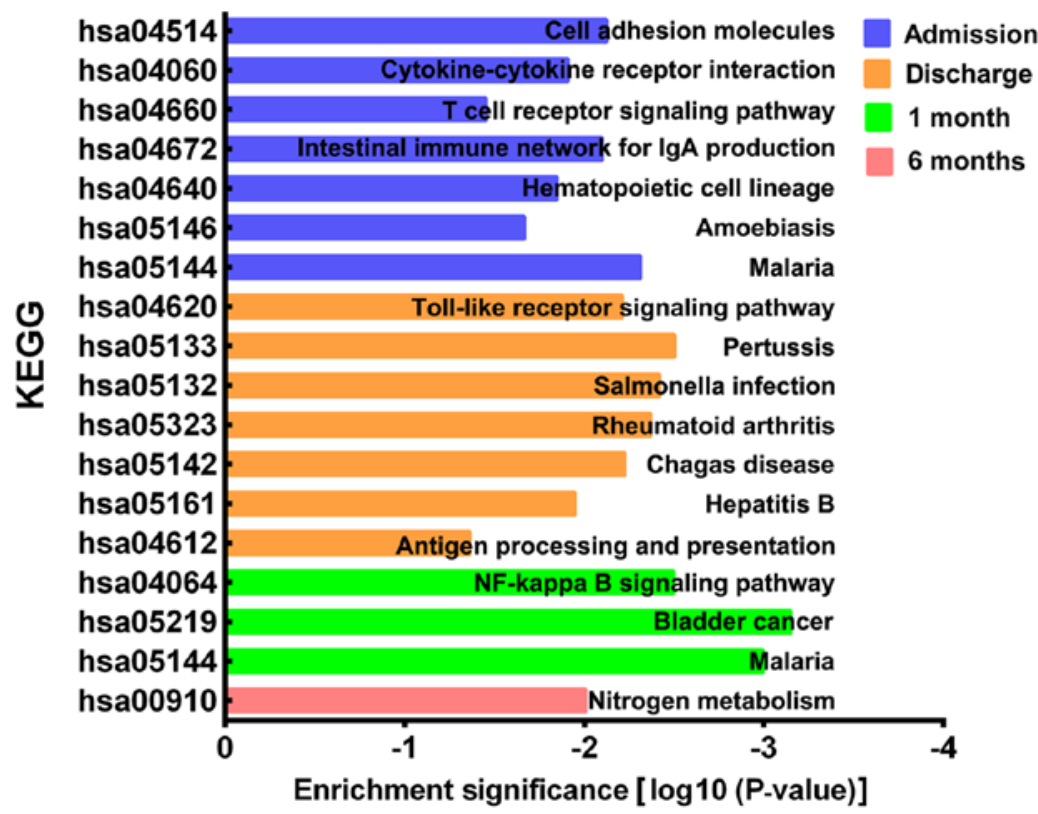

Figure 4. Significant KEGG pathways enriched by time-series differential expressed genes at different time points. KEGG, Kyoto Encyclopedia of Genes and Genomes.

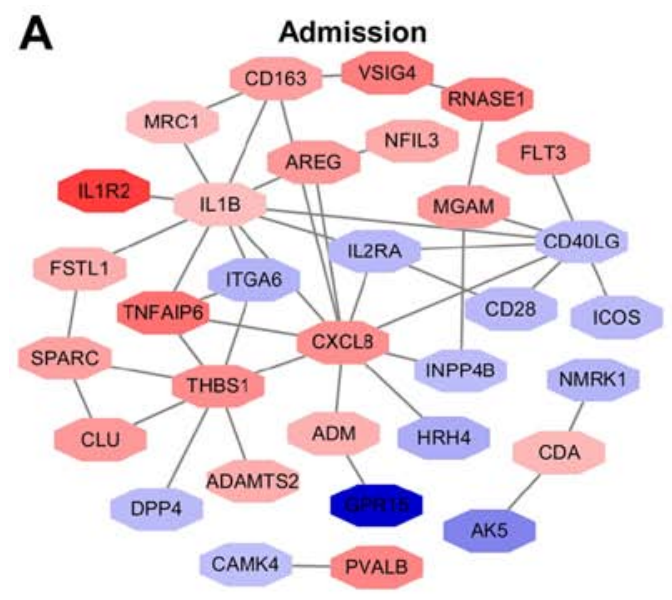

B
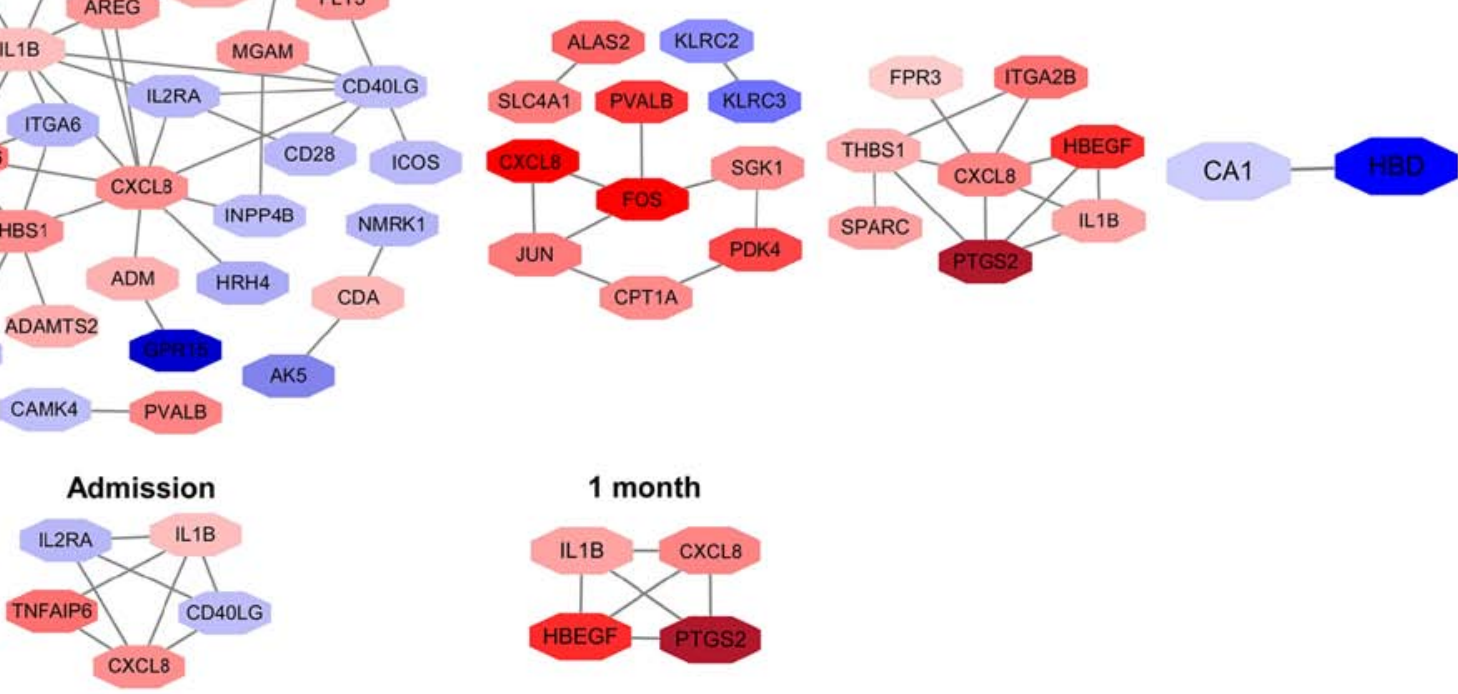

1 month

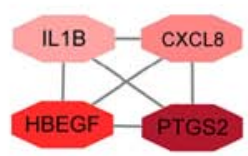

Figure 5. (A) Protein-protein interaction network and (B) module network analysis of DEGs at different time points. Red nodes represent upregulated DEGs and blue nodes represent downregulated DEGs. DEGs, differentially expressed genes.

expression of AK5 in the blood of patients with HF may be an adaptive response of the brain to ischemic stress present in the heart. Future studies are required to elucidate the role of AK5 in the development of HF following AMI.

Prolonged coronary occlusion leads to the death of cardiomyocytes and damage of the extracellular matrix in the tissues, triggering activation of an intense inflammatory response (43). The inflammatory response following AMI may aid in the repair of the infarcted myocardium through scar formation to maintain cardiac integrity; however, excessive inflammatory response may contribute to adverse left ventricle remodeling and HF (44). Both human and animal studies demonstrated that the inflammatory response is involved in the pathogenesis of HF $(45,46)$. Compared with the healthy controls, patients with HF exhibited increased levels of circulating inflammatory cytokines, including tumor necrosis factor (TNF)- $\alpha$, C-reactive protein, IL1 $\beta$ and IL6 (47-49). Previous studies suggested that inhibition of IL1 with anakinra or anti-IL1 $\beta$ antibodies may reduce chamber dilation and improve cardiac dysfunction (50,51). Furthermore, a clinical trial reported that inhibition of TNF- $\alpha$ with a recombinant chimeric soluble TNF receptor type 2 had beneficial effects on cardiac function in 
patients with chronic HF (52). The aforementioned studies suggested that the inflammatory response may be implicated in the development of HF following AMI.

The immune response was revealed to participate in positive and negative ventricle remodeling following MI $(53,54)$. The immune response may synergistically function with the inflammatory response to repair the infarcted myocardium by removing dead cardiomyocytes and matrix debris, and producing mediators that activate fibroblast growth and angiogenesis (55). However, an overactive immune response may increase dilative remodeling and result in chamber dilation, systolic dysfunction and HF in patients surviving AMI (56). A double blind, placebo-controlled study of intravenous immunoglobulin induction and maintenance therapy for 26 weeks resulted in a 5\% improvement of LVEF independent of the etiology of HF in 40 patients with ischemic and non-ischemic cardiomyopathy (57). Animal experiments revealed that regulation of the immune response by targeting interferon regulatory factors may regulate cardiac hypertrophy in vitro and in vivo $(58,59)$. These studies demonstrate the potential role of the immune response in the development of HF following AMI.

Toll-like receptors are transmembrane proteins that are part of the innate immune system used to identify pathogens and sense endogenous danger-associated molecular patterns released from necrotic or dying cells (60). Toll-like receptor 4 , one of the 13 known mammalian toll-like receptors, may mediate the inflammatory response in the infarcted heart (44). In a rat model of HF following AMI, the expression level of toll-like receptor 4 was found to be upregulated in chronic HF, and blockade of toll-like receptor 4 improved left ventricle function (61). In addition, the activation of toll-like receptors triggered a downstream signaling cascade involving the activation of NF- $\kappa \beta$, interferon regulatory factors, transcription factors and activator protein 1 , leading to the enhanced expression of a number of inflammatory genes (62). Therefore, the pathways of toll-like receptor signaling and NF- $\kappa \beta$ signaling, which were enriched in the current study, may be implicated in the development of HF following AMI.

CXCL8 and IL1 $\beta$ were hub genes analyzed by the PPI network. CXCL8 belongs to the CXC chemokine family and serves an important role in mediating neutrophil invasion in a number of inflammatory processes (63). The Controlled Rosuvastatin Multinational Trial in Heart Failure study revealed that increased expression levels of CXCL8 were associated with adverse outcome in patients with chronic HF (64). Husebye et al (65) demonstrated that high levels of CXCL8 in patients with ST-elevation myocardial infarction complicated with HF were associated with poor recovery of left ventricular function. IL1 $\beta$ is a member of the IL1 cytokine family and serves important roles in the regulation of cardiac inflammation and repair (43). IL1 $\beta$ promotes cardiac fibrosis by secreting cytokines, chemokines and matrix metalloproteinases (66). Furthermore, increased plasma IL1 $\beta$ levels have been associated with impaired myocardial function and left ventricle hypertrophy following reperfusion after MI (67). Based on the results obtained in the aforementioned studies, the upregulated expression levels of CXCL8 and IL1 $\beta$ may contribute to the development of HF following AMI.

In the present study, time-series DEGs between peripheral blood samples obtained from HF and non-HF patients were identified. A number of DEGs, including FADS2, LRRN3, GPR15, AK5, CXCL8 and IL1 $\beta$, were identified as possible target genes for the development of HF following AMI. Furthermore, the present study revealed that biological processes involved in the inflammatory and immune responses and the toll-like receptor and NF- $\kappa \beta$ signaling pathways may serve important roles in the development of HF following AMI. However, future experimental studies are required to substantiate the results obtained in the present study.

\section{Acknowledgements}

Not applicable.

\section{Funding}

No funding was received.

\section{Availability of data and materials}

The datasets generated and/or analyzed during the present study are available in the National Center of Biotechnology Information's GEO database (www.ncbi.nlm.nih.gov/geo) with the accession number GSE59867.

\section{Authors' contributions}

$\mathrm{XL}$ and $\mathrm{HJ}$ conceived and designed the study. BL and $\mathrm{XL}$ performed the data analysis. XL, BL and HJ wrote the paper. All authors approved the final manuscript.

\section{Ethics approval and consent to participate}

Not applicable.

\section{Patient consent for publication}

Not applicable.

\section{Competing interests}

The authors declare that they have no competing interests.

\section{References}

1. Roger VL: Epidemiology of heart failure. Circ Res 113: 646-659, 2013.

2. Roger VL, Weston SA, Redfield MM, Hellermann-Homan JP, Killian J, Yawn BP and Jacobsen SJ: Trends in heart failure incidence and survival in a community-based population. JAMA 292: 344-350, 2004.

3. Hoydal MA, Kirkeby-Garstad I, Karevold A, Wiseth R, Haaverstad R, Wahba A, Stølen TL, Contu R, Condorelli G, Ellingsen $\varnothing$, et al: Human cardiomyocyte calcium handling and transverse tubules in mid-stage of post-myocardial-infarction heart failure. ESC Heart Fail 5: 332-342, 2018.

4. McMurray JJ, Adamopoulos S, Anker SD, Auricchio A, Böhm M, Dickstein K, Falk V, Filippatos G, Fonseca C, Gomez-Sanchez MA, et al: ESC Guidelines for the diagnosis and treatment of acute and chronic heart failure 2012: The Task Force for the Diagnosis and Treatment of Acute and Chronic Heart Failure 2012 of the European Society of Cardiology. Developed in collaboration with the Heart Failure Association (HFA) of the ESC. Eur Heart J 33: 1787-1847, 2012. 
5. Hellermann JP, Jacobsen SJ, Gersh BJ, Rodeheffer RJ, Reeder GS and Roger VL: Heart failure after myocardial infarction: A review. Am J Med 113: 324-330, 2002.

6. Borghi C, Bacchelli S, Degli Esposti D and Ambrosioni E; Survival of Myocardial Infarction Long-Term Evaluation Study: Effects of early angiotensin-converting enzyme inhibition in patients with non-ST-elevation acute anterior myocardial infarction. Am Heart J 152: 470-477, 2006.

7. O'Connor CM, Hathaway WR, Bates ER, Leimberger JD, Sigmon KN, Kereiakes DJ, George BS, Samaha JK, AbbottsmithCW, Candela RJ, et al: Clinical characteristics and long-term outcome of patients in whom congestive heart failure develops after thrombolytic therapy for acute myocardial infarction: Development of a predictive model. Am Heart J 133: 663-673, 1997.

8. de Lemos JA, Morrow DA, Bentley JH, Omland T, Sabatine MS, McCabe $\mathrm{CH}$, Hall C, Cannon $\mathrm{CP}$ and Braunwald E: The prognostic value of B-type natriuretic peptide in patients with acute coronary syndromes. N Engl J Med 345: 1014-1021, 2001.

9. Haeck JD, Verouden NJ, Kuijt WJ, Koch KT, Van Straalen JP, Fischer J, Groenink M, Bilodeau L, Tijssen JG, Krucoff MW and De Winter RJ: Comparison of usefulness of N-terminal pro-brain natriuretic peptide as an independent predictor of cardiac function among admission cardiac serum biomarkers in patients with anterior wall versus nonanterior wall ST-segment elevation myocardial infarction undergoing primary percutaneous coronary intervention. Am J Cardiol 105: 1065-1069, 2010

10. Tousoulis D, Kampoli AM, Stefanadi E, Antoniades C, Siasos G Papavassiliou AG and Stefanadis C: New biochemical markers in acute coronary syndromes. Curr Med Chem 15: 1288-1296, 2008

11. Moe KT and Wong P: Current trends in diagnostic biomarkers of acute coronary syndrome. Ann Acad Med Singapore 39: 210-215, 2010.

12. Hall PA, Reis-Filho JS, Tomlinson IP and Poulsom R: An introduction to genes, genomes and disease. J Pathol 220: 109-113, 2010.

13. Heidecker B and Hare JM: The use of transcriptomic biomarkers for personalized medicine. Heart Fail Rev 12: 1-11, 2007.

14. Gurwitz D: Expression profiling: A cost-effective biomarker discovery tool for the personal genome era. Genome Med 5: 41, 2013.

15. Maciejak A, Kiliszek M, Michalak M, Tulacz D, Opolski G Matlak K, Dobrzycki S, Segiet A, Gora M and Burzynska B: Gene expression profiling reveals potential prognostic biomarkers associated with the progression of heart failure. Genome Med 7: 26, 2015.

16. Gautier L, Cope L, Bolstad BM and Irizarry RA: Affy-analysis of Affymetrix GeneChip data at the probe level. Bioinformatics 20: 307-315, 2004.

17. Ritchie ME, Phipson B, Wu D, Hu Y, Law CW, Shi W and Smyth GK: limma powers differential expression analyses for RNA-sequencing and microarray studies. Nucleic Acids Res 43 : e47, 2015 .

18. Sturn A, Quackenbush J and Trajanoski Z: Genesis: Cluster analysis of microarray data. Bioinformatics 18: 207-208, 2002.

19. Dennis G Jr, Sherman BT, Hosack DA, Yang J, Gao W, Lane HC and Lempicki RA: DAVID: Database for Annotation, Visualization, and Integrated Discovery. Genome Biol 4: P3, 2003.

20. Stelzl U, Worm U, Lalowski M, Haenig C, Brembeck FH, Goehler H, Stroedicke M, Zenkner M, Schoenherr A, Koeppen S, et al: A human protein-protein interaction network: A resource for annotating the proteome. Cell 122: 957-968,2005.

21. Franceschini A, Szklarczyk D, Frankild S, Kuhn M, Simonovic M, Roth A, Lin J, Minguez P, Bork P, von Mering C and Jensen LJ: STRING v9.1: Protein-protein interaction networks, with increased coverage and integration. Nucleic Acids Res 41: D808-D815, 2013.

22. Shannon P, Markiel A, Ozier O, Baliga NS, Wang JT, Ramage D, Amin N, Schwikowski B and Ideker T: Cytoscape: A software environment for integrated models of biomolecular interaction networks. Genome Res 13: 2498-2504, 2003.

23. Bader GD and Hogue CW: An automated method for finding molecular complexes in large protein interaction networks. BMC Bioinformatics 4: 2, 2003.

24. Maere S, Heymans K and Kuiper M: BiNGO: A Cytoscape plugin to assess overrepresentation of gene ontology categories in biological networks. Bioinformatics 21: 3448-3449, 2005.

25. Li SW, Lin K, Ma P, Zhang ZL, Zhou YD, Lu SY, Zhou X and Liu SM: FADS gene polymorphisms confer the risk of coronary artery disease in a Chinese Han population through the altered desaturase activities: Based on high-resolution melting analysis. PLoS One 8: e55869, 2013
26. Rupp H, Rupp TP, Alter P and Maisch B: N-3 polyunsaturated fatty acids and statins in heart failure. Lancet 373: 378-380, 2009

27. Rupp H, Rupp TP, Alter P and Maisch B: Inverse shift in serum polyunsaturated and monounsaturated fatty acids is associated with adverse dilatation of the heart. Heart 96: 595-598, 2010.

28. Rupp H, Rupp TP, Alter P and Maisch B: Mechanisms involved in the differential reduction of omega- 3 and omega- 6 highly unsaturated fatty acids by structural heart disease resulting in 'HUFA deficiency'. Can J Physiol Pharmacol 90: 55-73, 2012.

29. Alter P, Gluck T, Figiel JH, Koczulla AR, Vogelmeier CF and Rupp H: From heart failure to highly unsaturated fatty acid deficiency and vice versa: Bidirectional heart and liver interactions. Can J Cardiol 32: 217-225, 2016.

30. Anderson JS, Nettleton JA, Hundley WG, Tsai MY, Steffen LM, Lemaitre RN, Siscovick D, Lima J, Prince MR and Herring ton D: Associations of plasma phospholipid omega-6 and omega-3 polyunsaturated Fatty Acid levels and MRI measures of cardiovascular structure and function: The multiethnic study of atherosclerosis. J Nutr Metab 2011: 315134, 2011.

31. Hamano S, Ohira M, Isogai E, Nakada K and Nakagawara A: Identification of novel human neuronal leucine-rich repeat (hNLRR) family genes and inverse association of expression of Nbla10449/hNLRR-1 and Nbla10677/hNLRR-3 with the prognosis of primary neuroblastomas. Int J Oncol 24: 1457-1466, 2004.

32. Fukamachi K, Matsuoka Y, Ohno H, Hamaguchi T and Tsuda H: Neuronal leucine-rich repeat protein-3 amplifies MAPK activation by epidermal growth factor through a carboxyl-terminal region containing endocytosis motifs. J Biol Chem 277: 43549-43552, 2002.

33. Yokota T and Wang Y: p38 MAP kinases in the heart. Gene 575: 369-376, 2016

34. Zhang Q, Lu L, Liang T, Liu M, Wang ZL and Zhang PY: MAPK pathway regulated the cardiomyocyte apoptosis in mice with post-infarction heart failure. Bratisl Lek Listy 118: 339-346, 2017.

35. Fei AH, Wang FC, Wu ZB and Pan SM: Phosphocreatine attenuates angiotensin II-induced cardiac fibrosis in rat cardiomyocytes through modulation of MAPK and NF- $\kappa \mathrm{B}$ pathway. Eur Rev Med Pharmacol Sci 20: 2726-2733, 2016.

36. Deng HK, Unutmaz D, KewalRamani VN and Littman DR: Expression cloning of new receptors used by simian and human immunodeficiency viruses. Nature 388: 296-300, 1997.

37. Nguyen LP, Pan J, Dinh TT, Hadeiba H, O'Hara E III, Ebtikar A, Hertweck A, Gökmen MR, Lord GM, Jenner RG, et al: Role and species-specific expression of colon T cell homing receptor GPR15 in colitis. Nat Immunol 16: 207-213, 2015.

38. Koks G, Uudelepp ML, Limbach M, Peterson P, Reimann E and Koks S: Smoking-induced expression of the GPR15 gene indicates its potential role in chronic inflammatory pathologies. Am J Pathol 185: 2898-2906, 2015.

39. Pan B, Wang X, Nishioka C, Honda G, Yokoyama A, Zeng L, $\mathrm{Xu} \mathrm{K}$ and Ikezoe T: G-protein coupled receptor 15 mediates angiogenesis and cytoprotective function of thrombomodulin. Sci Rep 7: 692, 2017

40. Dzeja P and Terzic A: Adenylate kinase and AMP signaling networks: Metabolic monitoring, signal communication and body energy sensing. Int J Mol Sci 10: 1729-1772, 2009.

41. Hardie DG and Hawley SA: AMP-activated protein kinase: The energy charge hypothesis revisited. Bioessays 23: 1112-1119, 2001.

42. Lai Y, Hu X, Chen G, Wang X and Zhu B: Down-regulation of adenylate kinase 5 in temporal lobe epilepsy patients and rat model. J Neurol Sci 366: 20-26, 2016.

43. Huang S and Frangogiannis NG: Anti-inflammatory therapies in myocardial infarction: Failures, hopes and challenges. Br J Pharmacol 175: 1377-1400, 2018.

44. Fang L, Moore XL, Dart AM and Wang LM: Systemic inflammatory response following acute myocardial infarction. J Geriatr Cardiol 12: 305-312, 2015.

45. Nakayama $\mathrm{H}$ and Otsu $\mathrm{K}$ : Translation of hemodynamic stress to sterile inflammation in the heart. Trends Endocrinol Metab 24: 546-553, 2013.

46. Yndestad A, Damas JK, Oie E, Ueland T, Gullestad L and Aukrust P: Systemic inflammation in heart failure-the whys and wherefores. Heart Fail Rev 11: 83-92, 2006.

47. Levine B, Kalman J, Mayer L, Fillit HM and Packer M: Elevated circulating levels of tumor necrosis factor in severe chronic heart failure. N Engl J Med 323: 236-241, 1990.

48. Pye M, Rae AP and Cobbe SM: Study of serum C-reactive protein concentration in cardiac failure. Br Heart J 63: 228-230, 1990. 
49. Testa M, Yeh M, Lee P, Fanelli R, Loperfido F, Berman JW and LeJemtel TH: Circulating levels of cytokines and their endogenous modulators in patients with mild to severe congestive heart failure due to coronary artery disease or hypertension. J Am Coll Cardiol 28: 964-971, 1996.

50. Abbate A, Salloum FN, Vecile E, Das A, Hoke NN, Straino S, Biondi-Zoccai GG, Houser JE, Qureshi IZ, Ownby ED, et al: Anakinra, a recombinant human interleukin-1 receptor antagonist, inhibits apoptosis in experimental acute myocardial infarction. Circulation 117: 2670-2683, 2008

51. Toldo S, Mezzaroma E, Van Tassell BW, Farkas D, Marchetti C, Voelkel NF and Abbate A: Interleukin-1 $\beta$ blockade improves cardiac remodelling after myocardial infarction without interrupting the inflammasome in the mouse. Exp Physiol 98: 734-745, 2013.

52. Deswal A, Bozkurt B, Seta Y, Parilti-Eiswirth S, Hayes FA, Blosch C and Mann DL: Safety and efficacy of a soluble P75 tumor necrosis factor receptor (Enbrel, etanercept) in patients with advanced heart failure. Circulation 99: 3224-3226, 1999.

53. Frieler RA and Mortensen RM: Immune cell and other noncardiomyocyte regulation of cardiac hypertrophy and remodeling. Circulation 131: 1019-1030, 2015.

54. Nahrendorf $M$ and Swirski FK: Innate immune cells in ischaemic heart disease: Does myocardial infarction beget myocardial infarction? Eur Heart J 37: 868-872, 2016.

55. Frangogiannis NG: The immune system and cardiac repair. Pharmacol Res 58: 88-111, 2008.

56. Frangogiannis NG: The immune system and the remodeling infarcted heart: Cell biological insights and therapeutic opportunities. J Cardiovasc Pharmacol 63: 185-195, 2014.

57. Gullestad L, Aass H, Fjeld JG, Wikeby L, Andreassen AK, Ihlen H, Simonsen S, Kjekshus J, Nitter-Hauge S, Ueland T, et al: Immunomodulating therapy with intravenous immunoglobulin in patients with chronic heart failure. Circulation 103: 220-225, 2001.

58. Jiang DS, Li L, Huang L, Gong J, Xia H, Liu X, Wan N, Wei X, Zhu X, Chen Y, et al: Interferon regulatory factor 1 is required for cardiac remodeling in response to pressure overload. Hypertension 64: 77-86, 2014.

59. Jiang DS, Wei X, Zhang XF, Liu Y, Zhang Y, Chen K, Gao L, Zhou H, Zhu XH, Liu PP, et al: IRF8 suppresses pathological cardiac remodelling by inhibiting calcineurin signalling. Nat Commun 5: 3303, 2014.
60. Yang Y, Lv J, Jiang S, Ma Z, Wang D, Hu W, Deng C, Fan C, Di S, Sun Y and Yi W: The emerging role of Toll-like receptor 4 in myocardial inflammation. Cell Death Dis 7: e2234, 2016.

61. Liu L, Wang Y, Cao ZY, Wang MM, Liu XM, Gao T, Hu QK, Yuan WJ and Lin L: Up-regulated TLR4 in cardiomyocytes exacerbates heart failure after long-term myocardial infarction. J Cell Mol Med 19: 2728-2740, 2015.

62. Mann DL, Topkara VK, Evans S and Barger PM: Innate immunity in the adult mammalian heart: For whom the cell tolls. Trans Am Clin Climatol Assoc 121: 34-51, 2010.

63. de Winter RJ, Manten A, de Jong YP, Adams R, van Deventer SJ and Lie KI: Interleukin 8 released after acute myocardial infarction is mainly bound to erythrocytes. Heart 78: 598-602, 1997.

64. Nymo SH, Hulthe J, Ueland T, McMurray J, Wikstrand J, Askevold ET, Yndestad A, Gullestad L and Aukrust P: Inflammatory cytokines in chronic heart failure: Interleukin- 8 is associated with adverse outcome. Results from CORONA. Eur J Heart Fail 16: 68-75, 2014.

65. Husebye T, Eritsland J, Arnesen H, Bjørnerheim R, Mangschau A, Seljeflot I and Andersen GØ: Association of interleukin 8 and myocardial recovery in patients with ST-elevation myocardial infarction complicated by acute heart failure. PLoS One 9: e112359, 2014.

66. Saxena A, Chen W, Su Y, Rai V, Uche OU, Li N and Frangogiannis NG: IL-1 induces proinflammatory leukocyte infiltration and regulates fibroblast phenotype in the infarcted myocardium. J Immunol 191: 4838-4848, 2013.

67. Orn S, Ueland T, Manhenke C, Sandanger $\varnothing$, Godang K, Yndestad A, Mollnes TE, Dickstein K and Aukrust P: Increased interleukin-1 $\beta$ levels are associated with left ventricular hypertrophy and remodelling following acute ST segment elevation myocardial infarction treated by primary percutaneous coronary intervention. J Intern Med 272: 267-276, 2012.

This work is licensed under a Creative Commons

Attribution-NonCommercial-NoDerivatives 4.0 International (CC BY-NC-ND 4.0) License. 